\title{
Generalized Jordan Derivations of Incidence Algebras
}

\author{
Ruth Nascimento Ferreira ${ }^{1}$ and Bruno Leonardo Macedo Ferreira ${ }^{2, *}$ \\ ${ }^{1}$ Universidade Tecnológica Federal do Paraná, Av. Profa. Laura Pacheco Bastos, 800, 85053-510, \\ Guarapuava, Brazil \\ e-mail: ruthnascimento@utfpr.edu.br \\ ${ }^{2}$ Universidade Tecnológica Federal do Paraná, Av. Profa. Laura Pacheco Bastos, 800, 85053-510, \\ Guarapuava, Brazil \\ e-mail: brunoferreira@utfpr.edu.br \\ * Corresponding author
}

\begin{abstract}
For a given ring $\Re$ and a locally finite pre-ordered set $(X, \leq)$, consider $I(X, \mathfrak{R})$ to be the incidence algebra of $X$ over $\Re$. Motivated by a Xiao's result which states that every Jordan derivation of $I(X, \mathfrak{R})$ is a derivation in the case $\mathfrak{R}$ is 2 -torsion free, one proves that each generalized Jordan derivation of $I(X, \Re)$ is a generalized derivation provided $\Re$ is 2 -torsion free, getting as a consequence the above mentioned result.
\end{abstract}

\section{Introduction}

For a given ring $\mathfrak{R}$, recall that a linear map $d$ from $\mathfrak{R}$ into itself is called a derivation if $d(a b)=d(a) b+a d(b)$ for all $a, b \in \mathfrak{R} ;$ and a Jordan derivation if $d\left(a^{2}\right)=$ $d(a) a+a d(a)$ for each $a \in \mathfrak{R}$. More generally [5], if there is a derivation $\tau: \mathfrak{R} \rightarrow \mathfrak{R}$ such that $d(a b)=d(a) b+a \tau(b)$ for all $a, b \in \mathfrak{R}$, then $d$ is called a generalized derivation and $\tau$ is the relating derivation; analogously, if there is a Jordan derivation

Received: August 3, 2019; Accepted: September 2, 2019

2010 Mathematics Subject Classification: 16W25, 47B47.

Keywords and phrases: generalized derivation, generalized Jordan derivation, incidence algebras.

Copyright (C) 2019 Ruth Nascimento Ferreira and Bruno Leonardo Macedo Ferreira. This is an open access article distributed under the Creative Commons Attribution License, which permits unrestricted use, distribution, and reproduction in any medium, provided the original work is properly cited. 
$\tau: \mathfrak{R} \rightarrow \mathfrak{R}$ such that $d\left(a^{2}\right)=d(a) a+a \tau(a)$ for all $a \in \mathfrak{R}$, then $d$ is called a generalized Jordan derivation and $\tau$ is the relating Jordan derivation. The structures of derivations, Jordan derivations, generalized derivations and generalized Jordan derivations were systematically studied. It is obvious that every generalized derivation is a generalized Jordan derivation and every derivation is a Jordan derivation. But the converse is in general not true. Herstein [4] showed that every Jordan derivation from a 2-torsion free prime ring into itself is a derivation. Brešar [2] proved that Herstein's result is true for 2-torsion free semiprime rings. Jing and $\mathrm{Lu}$, motivated by the concept of generalized derivation, introduce this concept of generalized Jordan derivation in [5].

Let us now recall the notion of incidence algebra [7], [12], which we deal in this paper. Let $(X, \leq)$ be a locally finite pre-ordered set. This means $\leq$ is a reflexive and transitive binary relation on the set $X$, and for any $x \leq y$ in $X$ there are only finitely many elements $z$ satisfying $x \leq z \leq y$. The incidence algebra $I(X, \mathfrak{R})$ of $X$ over $\mathfrak{R}$ is defined as the set

$$
I(X, \mathfrak{R}):=\{f: X \times X \rightarrow \mathfrak{R} \mid f(x, y)=0 \text { if } x \nless y\}
$$

with algebraic operation given by

$$
\begin{gathered}
(f+g)(x, y)=f(x, y)+g(x, y), \\
(r f)(x, y)=r f(x, y), \\
(f g)(x, y)=\sum_{x \leq z \leq y} f(x, z) g(z, y)
\end{gathered}
$$

for all $f, g \in I(X, \mathfrak{R}), r \in \mathfrak{R}$ and $x, y, z \in X$. The product $f g$ is usually called convolution in function theory. It would be helpful to point out that the full matrix algebra $M_{n}(\mathfrak{R})$ and the upper (or lower) triangular matrix algebras $T_{n}(\mathfrak{R})$ are special examples of incidence algebras. The identity element $\delta$ of $I(X, \mathfrak{R})$ is given by $\delta(x, y)=\delta_{x y}$ for $x \leq y$, where $\delta_{x y} \in\{0,1\}$ is the Kronecker delta. For given $x, y \in X$ with $x \leq y$, let $e_{x y}$ be defined by $e_{x y}(u, v)=1$ if $(u, v)=(x, y)$, and $e_{x y}(u, v)=0$ otherwise. Then $e_{x y} e_{u v}=\delta_{y u} e_{x v}$ by the definition of convolution. Moreover, the set $B:=\left\{e_{x y} \mid x \leq y\right\}$ forms an $\mathfrak{R}$-linear basis of $I(X, \mathfrak{R})$. Note that 
incidence algebras allow infinite summation, and hence the $\mathfrak{R}$-linear map here means a map preserving in finite sum and scalar multiplication.

Incidence algebras were first considered by Ward [15] as generalized algebras of arithmetic functions. Rota and Stanley developed incidence algebras as the fundamental structures of enumerative combinatorial theory and allied areas of arithmetic function theory (see [11]). Motivated by the results of Stanley [13], automorphisms and other algebraic mappings of incidence algebras have been extensively studied (see [1], [3], [6], [7], [8], [9], [10], [11] and the references therein). Baclawski [1] studied the automorphisms and derivations of incidence algebras $I(X, \mathfrak{R})$ when $X$ is a locally finite partially ordered set. More specifically, he proved that every derivation of $I(X, \mathfrak{R})$ with $X$ a locally finite partially ordered set can be decomposed as a sum of an inner derivation and a transitive induced derivation. Koppinen [7] has extended these results to the incidence algebras $I(X, \mathfrak{R})$ with $X$ a locally finite pre-ordered set. Xiao [14] proved that every Jordan derivation of $I(X, \mathfrak{R})$ is a derivation provided that $\mathfrak{R}$ is 2 -torsion free. Motivated by Xiao's result our main objective is to prove that every generalized Jordan derivation of $I(X, \mathfrak{R})$ is a generalized derivation provided that $\mathfrak{R}$ is 2 -torsion free.

\section{Results}

We first collect some background material to prove our main result. Throughout this section, $\mathfrak{R}$ denotes a 2-torsion free ring. Let $\Xi: I(X, \mathfrak{R}) \rightarrow I(X, \mathfrak{R})$ be a generalized Jordan derivation and $\tau: I(X, \mathfrak{R}) \rightarrow I(X, \mathfrak{R})$ the relating Jordan derivation.

Lemma 2.1. For all $a, b, c \in I(X, \mathfrak{R})$, the following statements hold:

(1) $\Xi(a b+b a)=\Xi(a) b+a \tau(b)+\Xi(b) a+b \tau(a)$,

(2) $\Xi(a b a)=\Xi(a) b a+a \tau(b) a+a b \tau(a)$,

(3) $\Xi(a b c+c b a)=\Xi(a) b c+a \tau(b) c+a b \tau(c)+\Xi(c) b a+c \tau(b) a+c b \tau(a)$.

Proof. See [5].

According to Lemma 2.1, $\Xi(a b a)=\Xi(a) b a+a \tau(b) a+a b \tau(a)$. In the case $a b=b a=0$, we obtain $a \tau(b) a=0$. Furthermore, it follows that 


$$
\Xi(e)=\Xi(e) e+e \tau(e)
$$

for any idempotent $e \in I(X, \Re)$. In particular, since (1), $e \tau(a) e=0$, for any $a \in I(X, \mathfrak{R}) \quad$ satisfying $\quad e a=a e=0, \quad$ and $\quad \Xi(a) e+a \tau(e)+\Xi(e) a+e \tau(a)=0$. Multiplying by $e$ on the right yields

$$
\Xi(a) e+a \tau(e)=0=\Xi(e) a+e \tau(a),
$$

for any idempotent $e$ satisfying $e a=a e=0$.

Now assume that the set $B:=\left\{e_{x y} \mid x \leq y\right\}$ forms an $\Re$-linear basis of $I(X, \mathfrak{R})$. It is a consequence of (1) that

$$
\Xi\left(e_{i i}\right)=\Xi\left(e_{i i}\right) e_{i i}+e_{i i} \tau\left(e_{i i}\right) \text { and } e_{k i} \tau\left(e_{i i}\right) e_{i j}=0,
$$

for all $i$ and $k \leq i \leq j$. From Lemma 2.1 and the fact that $\Xi\left(e_{i j}\right)=\Xi\left(e_{i i} e_{i j}+e_{i j} e_{i i}\right)$ for all $1 \leq i<j \leq n$, we obtain

$$
\Xi\left(e_{i j}\right)=\Xi\left(e_{i i}\right) e_{i j}+e_{i i} \tau\left(e_{i j}\right)+\Xi\left(e_{i j}\right) e_{i i}+e_{i j} \tau\left(e_{i i}\right)
$$

whenever $i<j$. Furthermore (2) implies that

$$
\Xi\left(e_{k j}\right) e_{i i}+e_{k j} \Xi\left(e_{i i}\right)=\Xi\left(e_{i i}\right) e_{k j}+e_{i i} \tau\left(e_{k j}\right)=0
$$

for all $k, j \neq i$. Define a $\mathfrak{R}$-linear map $\phi$ from $I(X, \mathfrak{R})$ into itself by letting

$$
\phi\left(e_{i j}\right)=\Xi\left(e_{i i}\right) e_{i j}+e_{i i} \tau\left(e_{i j}\right), \quad i \leq j \text {. }
$$

According to (3), $\phi\left(e_{i i}\right)=\Xi\left(e_{i i}\right)$. Xiao proved the following result.

Lemma 2.2 (Lemma 3.2 [14]). Let $\tau: I(X, \mathfrak{R}) \rightarrow I(X, \mathfrak{R})$ be a Jordan derivation. Then

$$
\tau\left(e_{i j}\right)=\sum_{x \in L_{i}} C_{x i}^{i i} e_{x j}+C_{i j}^{i j} e_{i j}+\sum_{y \in R_{j}} C_{j y}^{j j} e_{i y}+C_{j i}^{i j} e_{j i}
$$

for all $e_{i j} \in B$, where the coefficients $C_{x y}^{i j}$ are subject to the following relations

$$
\begin{gathered}
C_{j k}^{j j}+C_{j k}^{k k}=0, \text { if } j \leq k ; \\
C_{i j}^{i j}+C_{j k}^{j k}=C_{i k}^{i k}, \text { if } i \leq j, j \leq k .
\end{gathered}
$$


Lemma 2.3. $\phi$ is a generalized derivation.

Proof. Let us consider $d\left(e_{i j}\right)=\sum_{x \in L_{i}} C_{x i}^{i i} e_{x j}+C_{i j}^{i j} e_{i j}+\sum_{y \in R_{j}} C_{j y}^{j j} e_{i y}$ for all $e_{i j} \in B$, where the coefficients $C_{x y}^{i j}$ are subject to the following relations

$$
\begin{gathered}
C_{j k}^{j j}+C_{j k}^{k k}=0, \quad \text { if } j \leq k ; \\
C_{i j}^{i j}+C_{j k}^{j k}=C_{i k}^{i k}, \quad \text { if } i \leq j, j \leq k .
\end{gathered}
$$

By [14, Theorem 2.2] $d$ is a derivation. First we check that

$$
\phi\left(e_{i j} e_{k l}\right)=\phi\left(e_{i j}\right) e_{k l}+e_{i j} d\left(e_{k l}\right),
$$

for all $e_{i j}, e_{k l} \in B$. We split the argument into two cases.

Case 1: $j \neq k$. Since $\phi\left(e_{i j} e_{k l}\right)=0$, it suffices to prove that $\phi\left(e_{i j}\right) e_{k l}+$ $e_{i j} d\left(e_{k l}\right)=0$. By (6) we get

$$
\begin{aligned}
\phi\left(e_{i j}\right) e_{k l}+e_{i j} d\left(e_{k l}\right) & =\left(\Xi\left(e_{i i}\right) e_{i j}+e_{i i} \tau\left(e_{i j}\right)\right) e_{k l}+e_{i j} d\left(e_{k l}\right) \\
& =e_{i i} \tau\left(e_{i j}\right) e_{k l}+e_{i j} d\left(e_{k l}\right) .
\end{aligned}
$$

If $i \neq k$, then

$$
\begin{aligned}
e_{i i} \tau\left(e_{i j}\right) e_{k l}+e_{i j} d\left(e_{k l}\right) & =e_{i i} \tau\left(e_{i j}\right) e_{k l}+e_{i j} d\left(e_{k k}\right) e_{k l} \\
& =e_{i i}\left(\tau\left(e_{i j}\right) e_{k k}+e_{i j} d\left(e_{k k}\right)\right) e_{k l} \\
& =e_{i i} 0 e_{k l} \\
& =0,
\end{aligned}
$$

by Lemma 2.2 and $\tau\left(e_{i j}\right) e_{k k}=\tau\left(e_{i j} e_{k k}\right)-e_{i j} \tau\left(e_{k k}\right)$. Finally, if $i=k$, then

$$
\begin{aligned}
e_{i i} \tau\left(e_{i j}\right) e_{i l}+e_{i j} d\left(e_{i l}\right) & =e_{i i} \tau\left(e_{i j}\right) e_{i l}+e_{i j} d\left(e_{i i} e_{i l}\right) \\
& =e_{i i} \tau\left(e_{i j}\right) e_{i l}+e_{i j} d\left(e_{i i}\right) e_{i l}
\end{aligned}
$$




$$
\begin{aligned}
= & \left(e_{i i} \tau\left(e_{i j}\right)+e_{i j} d\left(e_{i i}\right) e_{i l}\right. \\
= & \left(\tau\left(e_{i j}\right)-\tau\left(e_{i i}\right) e_{i j}\right. \\
& \left.-\tau\left(e_{i j}\right) e_{i i}-e_{i j} \tau\left(e_{i i}\right)+e_{i j} d\left(e_{i i}\right)\right) e_{i l} \\
= & e_{i j}\left(d\left(e_{i i}\right)-\tau\left(e_{i i}\right)\right) e_{i l}=0 .
\end{aligned}
$$

Case 2: $j=k$. We must prove that

$$
\phi\left(e_{i l}\right)=\phi\left(e_{i j}\right) e_{j l}+e_{i j} d\left(e_{j l}\right) .
$$

Assume $i<j<l$. As a consequence of (6),

$$
\begin{aligned}
\phi\left(e_{i j}\right) e_{j l}+e_{i j} d\left(e_{j l}\right) & =\left(\Xi\left(e_{i i}\right) e_{i j}+e_{i i} \tau\left(e_{i j}\right)\right) e_{j l}+e_{i j} d\left(e_{j l}\right) \\
& =\phi\left(e_{i l}\right)-e_{i i}\left(\tau\left(e_{i l}\right)-\tau\left(e_{i j}\right) e_{j l}-e_{i j} d\left(e_{j l}\right)\right) \\
& =\phi\left(e_{i l}\right)-e_{i i}\left(e_{i j} \tau\left(e_{j l}\right)+\tau\left(e_{j l}\right) e_{i j}+e_{j l} \tau\left(e_{i j}\right)-e_{i j} d\left(e_{j l}\right)\right) \\
& =\phi\left(e_{i l}\right)-e_{i j}\left(\tau\left(e_{j l}\right)-d\left(e_{j l}\right)\right)=\phi\left(e_{i l}\right) .
\end{aligned}
$$

If $i=j<l$, then

$$
\begin{aligned}
\phi\left(e_{i i}\right) e_{i l}+e_{i i} d\left(e_{i l}\right) & =\Xi\left(e_{i i}\right) e_{i l}+e_{i i} \tau\left(e_{i l}\right)+e_{i i} d\left(e_{i l}\right)-e_{i i} \tau\left(e_{i l}\right) \\
& =\Xi\left(e_{i i}\right) e_{i l}+e_{i i} \tau\left(e_{i l}\right)=\phi\left(e_{i l}\right) .
\end{aligned}
$$

If $i<j=l$, then

$$
\begin{aligned}
\phi\left(e_{i j}\right) e_{j j}+e_{i j} d\left(e_{j j}\right)= & \left(\Xi\left(e_{i i}\right) e_{i j}+e_{i i} \tau\left(e_{i j}\right)\right) e_{j j}+e_{i j} d\left(e_{j j}\right) \\
= & \Xi\left(e_{i i}\right) e_{i j}+e_{i i} \tau\left(e_{i j}\right)+e_{i i} \tau\left(e_{i j}\right) e_{j j} \\
& -e_{i i} \tau\left(e_{i j}\right)+e_{i j} d\left(e_{j j}\right) .
\end{aligned}
$$

Since $e_{i i} \tau\left(e_{i j}\right) e_{j j}=C_{i j}^{i j} e_{j j}$,

$$
e_{i i} \tau\left(e_{i j}\right)=C_{i j}^{i j} e_{i j}+\sum_{y \in R_{j}} C_{j y}^{j j} e_{i y}
$$


and

$$
e_{i j} d\left(e_{j j}\right)=C_{j j}^{i j} e_{i j}+\sum_{y \in R_{j}} C_{j y}^{j j} e_{i y}
$$

it follows that $e_{i i} \tau\left(e_{i j}\right) e_{j j}-e_{i i} \tau\left(e_{i j}\right)+e_{i j} d\left(e_{j j}\right)=0$. Hence $\phi\left(e_{i j}\right) e_{j j}+e_{i j} d\left(e_{j j}\right)=$ $\Xi\left(e_{i i}\right) e_{i j}+e_{i i} \tau\left(e_{i j}\right)=\phi\left(e_{i j}\right)$. If $i=j=l$, by (3) we obtain $\phi\left(e_{i i}\right)=\Xi\left(e_{i i}\right)=$ $\Xi\left(e_{i i}\right) e_{i i}+e_{i i} \tau\left(e_{i i}\right)=\phi\left(e_{i i}\right) e_{i i}+e_{i i} d\left(e_{i i}\right)$. Thus, for all $e_{i j}, e_{k l} \in B$, we get $\phi\left(e_{i j} e_{k l}\right)=$ $\phi\left(e_{i j}\right) e_{k l}+e_{i j} d\left(e_{k l}\right)$. Finally, linearity of $\phi$ yields $\phi(a b)=\phi(a) b+a d(b)$ for all $a, b \in I(X, \mathfrak{R})$, which proves that $\phi$ is a generalized derivation.

We are now in a position to prove the main result of this paper.

Theorem 2.1. Let $\mathfrak{R}$ be a 2-torsion free commutative ring with identity. Then any generalized Jordan derivation of the incidence algebra $I(X, \mathfrak{R})$ is a generalized derivation.

Proof. Put $\Psi=\Xi-\phi$, then $\Psi\left(e_{i j}\right)=\Xi\left(e_{i j}\right)-\phi\left(e_{i j}\right)$ and $\Psi\left(e_{i i}\right)=\Xi\left(e_{i i}\right)-\phi\left(e_{i i}\right)$ $=0$ for all $e_{i i} \in B$. Since $\Psi$ is a generalized Jordan derivation, $\Psi\left(e_{i j}\right)=$ $\Psi\left(e_{i j} e_{j j}+e_{j j} e_{i j}\right)=\Psi\left(e_{i j}\right) e_{j j}+\Psi\left(e_{j j}\right) e_{i j}=\Psi\left(e_{i j}\right) e_{j j}$. According to (4) and (6), if $i<j$ we have

$$
\begin{aligned}
\Psi\left(e_{i j}\right) & =\Xi\left(e_{i j}\right) e_{i i}+e_{i j} \tau\left(e_{i i}\right) \\
& =\left(\phi\left(e_{i j}\right)+\Psi\left(e_{i j}\right)\right) e_{i i}+e_{i j} \tau\left(e_{i i}\right) \\
& =\phi\left(e_{i j}\right) e_{i i}+e_{i j} \tau\left(e_{i i}\right)+\Psi\left(e_{i j}\right) e_{i i} \\
& =\phi\left(e_{i j} e_{i i}\right)+\Psi\left(e_{i j}\right) e_{i i} \\
& =\Psi\left(e_{i j}\right) e_{i i} .
\end{aligned}
$$

Thus $\Psi\left(e_{i j}\right)=\Psi\left(e_{i j}\right) e_{j j}=0$. Therefore $\Psi=\Xi-\phi=0$ and $\Xi$ is a generalized derivation.

As a consequence of our Theorem we have the following result.

Corollary 2.1 (Theorem 3.3 [14]). Let $\Re$ be a 2-torsion free commutative ring with identity. Then every Jordan derivation of the incidence algebra $I(X, \Re)$ is a derivation. 


\section{References}

[1] K. Baclawski, Automorphisms and derivations of incidence algebras, Proc. Amer. Math. Soc. 36 (1972), 351-356. https://doi.org/10.1090/S0002-9939-1972-0313133-8

[2] M. Brešar, Jordan derivations on semiprime rings, Proc. Amer. Math. Soc. 104 (1988) 1003-1006. https://doi.org/10.1090/S0002-9939-1988-0929422-1

[3] S. P. Coelho and C. P. Milies, Derivations of upper triangular matrix rings, Linear Algebra Appl. 187 (1993), 263-267. https://doi.org/10.1016/0024-3795(93)90141-A

[4] I. N. Herstein, Jordan derivations of prime rings, Proc. Amer. Math. Soc. 8 (1957) 11041110. https://doi.org/10.1090/S0002-9939-1957-0095864-2

[5] W. Jing and S. Lu, Generalized Jordan derivations on prime rings and standard operator algebras, Taiwanese J. Math. 7 (2003) 605-613.

https://doi.org/10.11650/twjm/1500407580

[6] S. Jøndrup, Automorphisms and derivations of upper triangular matrix rings, Linear Algebra Appl. 221 (1995), 205-218. https://doi.org/10.1016/0024-3795(93)00255-X

[7] M. Koppinen, Automorphisms and higher derivations of incidence algebras. J. Algebra 174 (1995) 698-723. https://doi.org/10.1006/jabr.1995.1147

[8] D. Mathis, Differential polynomial rings and Morita equivalence, Comm. Algebra 10 (1982), 2001-2017. https://doi.org/10.1080/00927878208822818

[9] A. Nowicki, Derivations of special subrings of matrix rings and regular graphs, Tsukuba J. Math. 7 (1983), 281-297. https://doi.org/10.21099/tkbjm/1496159826

[10] A. Nowicki and I. Nowosad, Local derivations of subrings of matrix rings, Acta Math. Hungar. 105 (2004), 145-150. https://doi.org/10.1023/B:AMHU.0000045539.32024.db

[11] E. Spiegel, On the automorphisms of incidence algebras, J. Algebra 239 (2001), 615-623. https://doi.org/10.1006/jabr.2000.8702

[12] E. Spiegel and C. J. O’Donnell, Incidence Algebras, New York, NY: Marcel Dekker, 1997.

[13] R. Stanley, Structure of incidence algebras and their automorphism groups, Bull. Amer. Math. Soc. 76 (1970), 1236-1239. https://doi.org/10.1090/S0002-9904-1970-12617-9

[14] Z. Xiao, Jordan derivations of incidence algebras, Rocky Mountain J. Math. 45 (2015), 1357-1368. https://doi.org/10.1216/RMJ-2015-45-4-1357

[15] M. Ward, Arithmetic functions on rings, Ann. Math. 38 (1937), 725-732. https://doi.org/10.2307/1968611 